\title{
Some algebraic identities on quadra Fibona-Pell integer sequence
}

\section{Arzu Özkoç*}

"Correspondence:

arzuozkoc@duzce.edu.tr

Department of Mathematics,

Faculty of Arts and Science, Düzce

University, Düzce, Turkey

\begin{abstract}
In this work, we define a quadra Fibona-Pell integer sequence

$W_{n}=3 W_{n-1}-3 W_{n-3}-W_{n-4}$ for $n \geq 4$ with initial values $W_{0}=W_{1}=0, W_{2}=1, W_{3}=3$, and we derive some algebraic identities on it including its relationship with Fibonacci and Pell numbers.
\end{abstract}

Keywords: Fibonacci numbers; Lucas numbers; Pell numbers; Binet's formula; binary linear recurrences

\section{Preliminaries}

Let $p$ and $q$ be non-zero integers such that $D=p^{2}-4 q \neq 0$ (to exclude a degenerate case). We set the sequences $U_{n}$ and $V_{n}$ to be

$$
\begin{aligned}
& U_{n}=U_{n}(p, q)=p U_{n-1}-q U_{n-2}, \\
& V_{n}=V_{n}(p, q)=p V_{n-1}-q V_{n-2}
\end{aligned}
$$

for $n \geq 2$ with initial values $U_{0}=0, U_{1}=1, V_{0}=2$, and $V_{1}=p$. The sequences $U_{n}$ and $V_{n}$ are called the (first and second) Lucas sequences with parameters $p$ and $q . V_{n}$ is also called the companion Lucas sequence with parameters $p$ and $q$.

The characteristic equation of $U_{n}$ and $V_{n}$ is $x^{2}-p x+q=0$ and hence the roots of it are $x_{1}=\frac{p+\sqrt{D}}{2}$ and $x_{2}=\frac{p-\sqrt{D}}{2}$. So their Binet formulas are

$$
U_{n}=\frac{x_{1}^{n}-x_{2}^{n}}{x_{1}-x_{2}} \quad \text { and } \quad V_{n}=x_{1}^{n}+x_{2}^{n}
$$

for $n \geq 0$. For the companion matrix $M=\left[\begin{array}{cc}p-q \\ 1\end{array}\right]$, one has

$$
\left[\begin{array}{c}
U_{n} \\
U_{n-1}
\end{array}\right]=M^{n-1}\left[\begin{array}{l}
1 \\
0
\end{array}\right] \text { and }\left[\begin{array}{c}
V_{n} \\
V_{n-1}
\end{array}\right]=M^{n-1}\left[\begin{array}{l}
p \\
2
\end{array}\right]
$$

for $n \geq 1$. The generating functions of $U_{n}$ and $V_{n}$ are

$$
U(x)=\frac{x}{1-p x+q x^{2}} \quad \text { and } \quad V(x)=\frac{2-p x}{1-p x+q x^{2}} .
$$

Fibonacci, Lucas, Pell, and Pell-Lucas numbers can be derived from (1). Indeed for $p=1$ and $q=-1$, the numbers $U_{n}=U_{n}(1,-1)$ are called the Fibonacci numbers (A000045 in

(C) 2015 Özkoç; licensee Springer. This article is distributed under the terms of the Creative Commons Attribution 4.0 International License (http://creativecommons.org/licenses/by/4.0/), which permits unrestricted use, distribution, and reproduction in any medium, provided you give appropriate credit to the original author(s) and the source, provide a link to the Creative Commons license, and indicate if changes were made. 
OEIS), while the numbers $V_{n}=V_{n}(1,-1)$ are called the Lucas numbers (A000032 in OEIS). Similarly, for $p=2$ and $q=-1$, the numbers $U_{n}=U_{n}(2,-1)$ are called the Pell numbers (A000129 in OEIS), while the numbers $V_{n}=V_{n}(2,-1)$ are called the Pell-Lucas (A002203 in OEIS) (companion Pell) numbers (for further details see [1-6]).

\section{Quadra Fibona-Pell sequence}

In [7], the author considered the quadra Pell numbers $D(n)$, which are the numbers of the form $D(n)=D(n-2)+2 D(n-3)+D(n-4)$ for $n \geq 4$ with initial values $D(0)=D(1)=$ $D(2)=1, D(3)=2$, and the author derived some algebraic relations on it.

In [8], the authors considered the integer sequence (with four parameters) $T_{n}=-5 T_{n-1}-$ $5 T_{n-2}+2 T_{n-3}+2 T_{n-4}$ with initial values $T_{0}=0, T_{1}=0, T_{2}=-3, T_{3}=12$, and they derived some algebraic relations on it.

In the present paper, we want to define a similar sequence related to Fibonacci and Pell numbers and derive some algebraic relations on it. For this reason, we set the integer sequence $W_{n}$ to be

$$
W_{n}=3 W_{n-1}-3 W_{n-3}-W_{n-4}
$$

for $n \geq 4$ with initial values $W_{0}=W_{1}=0, W_{2}=1, W_{3}=3$ and call it a quadra FibonaPell sequence. Here one may wonder why we choose this equation and call it a quadra Fibona-Pell sequence. Let us explain: We will see below that the roots of the characteristic equation of $W_{n}$ are the roots of the characteristic equations of both Fibonacci and Pell sequences. Indeed, the characteristic equation of (3) is $x^{4}-3 x^{3}+3 x+1=0$ and hence the roots of it are

$$
\alpha=\frac{1+\sqrt{5}}{2}, \quad \beta=\frac{1-\sqrt{5}}{2}, \quad \gamma=1+\sqrt{2} \quad \text { and } \quad \delta=1-\sqrt{2} \text {. }
$$

(Here $\alpha, \beta$ are the roots of the characteristic equation of Fibonacci numbers and $\gamma, \delta$ are the roots of the characteristic equation of Pell numbers.) Then we can give the following results for $W_{n}$.

Theorem 1 The generating function for $W_{n}$ is

$$
W(x)=\frac{x^{2}}{x^{4}+3 x^{3}-3 x+1} .
$$

Proof The generating function $W(x)$ is a function whose formal power series expansion at $x=0$ has the form

$$
W(x)=\sum_{n=0}^{\infty} W_{n} x^{n}=W_{0}+W_{1} x+W_{2} x^{2}+\cdots+W_{n} x^{n}+\cdots
$$

Since the characteristic equation of (3) is $x^{4}-3 x^{3}+3 x+1=0$, we get

$$
\begin{aligned}
\left(1-3 x+3 x^{3}+x^{4}\right) W(x) & =\left(1-3 x+3 x^{3}+x^{4}\right)\left(W_{0}+W_{1} x+\cdots+W_{n} x^{n}+\cdots\right) \\
& =W_{0}+\left(W_{1}-3 W_{0}\right) x+\left(W_{2}-3 W_{1}\right) x^{2}
\end{aligned}
$$




$$
\begin{aligned}
& +\left(W_{3}-3 W_{2}+3 W_{0}\right) x^{3}+\cdots \\
& +\left(W_{n}-3 W_{n-1}+3 W_{n-3}+W_{n-4}\right) x^{n}+\cdots
\end{aligned}
$$

Notice that $W_{0}=W_{1}=0, W_{2}=1, W_{3}=3$, and $W_{n}=3 W_{n-1}-3 W_{n-3}-W_{n-4}$. So $(1-3 x+$ $\left.3 x^{3}+x^{4}\right) W(x)=x^{2}$ and hence the result is obvious.

Theorem 2 The Binet formula for $W_{n}$ is

$$
W_{n}=\left(\frac{\gamma^{n}-\delta^{n}}{\gamma-\delta}\right)-\left(\frac{\alpha^{n}-\beta^{n}}{\alpha-\beta}\right)
$$

for $n \geq 0$.

Proof Note that the generating function is $W(x)=\frac{x^{2}}{x^{4}+3 x^{3}-3 x+1}$. It is easily seen that $x^{4}+$ $3 x^{3}-3 x+1=\left(1-x-x^{2}\right)\left(1-2 x-x^{2}\right)$. So we can rewrite $W(x)$ as

$$
W(x)=\frac{x}{1-2 x-x^{2}}-\frac{x}{1-x-x^{2}}
$$

From (2), we see that the generating function for Pell numbers is

$$
P(x)=\frac{x}{1-2 x-x^{2}}
$$

and the generating function for the Fibonacci numbers is

$$
F(x)=\frac{x}{1-x-x^{2}}
$$

From (5), (6), (7), we get $W(x)=P(x)-F(x)$. So $W_{n}=\left(\frac{\gamma^{n}-\delta^{n}}{\gamma-\delta}\right)-\left(\frac{\alpha^{n}-\beta^{n}}{\alpha-\beta}\right)$ as we wanted.

The relationship with Fibonacci, Lucas, and Pell numbers is given below.

Theorem 3 For the sequences $W_{n}, F_{n}, L_{n}$, and $P_{n}$, we have:

(1) $W_{n}=P_{n}-F_{n}$ for $n \geq 0$.

(2) $W_{n+1}+W_{n-1}=\left(\gamma^{n}+\delta^{n}\right)-\left(\alpha^{n}+\beta^{n}\right)$ for $n \geq 1$.

(3) $\sqrt{5} F_{n}+2 \sqrt{2} P_{n}=\left(\gamma^{n}-\delta^{n}\right)+\left(\alpha^{n}-\beta^{n}\right)$ for $n \geq 1$.

(4) $L_{n}+P_{n+1}+P_{n-1}=\alpha^{n}+\beta^{n}+\gamma^{n}+\delta^{n}$ for $n \geq 1$.

(5) $2\left(W_{n+1}-W_{n}+F_{n-1}\right)=\gamma^{n}+\delta^{n}$ for $n \geq 1$.

(6) $\lim _{n \rightarrow \infty} \frac{W_{n}}{W_{n-1}}=\gamma$.

Proof (1) It is clear from the above theorem, since $W(x)=P(x)-F(x)$.

(2) Since $6 W_{n-1}+W_{n+2}=3 W_{n+1}-3 W_{n-1}-W_{n-2}+6 W_{n-1}$, we get

$$
\begin{aligned}
W_{n+1}+W_{n-1}= & 2 W_{n-1}+\frac{1}{3} W_{n-2}+\frac{1}{3} W_{n+2} \\
= & \frac{6}{3}\left(\frac{\gamma^{n-1}-\delta^{n-1}}{\gamma-\delta}-\frac{\alpha^{n-1}-\beta^{n-1}}{\alpha-\beta}\right) \\
& +\frac{1}{3}\left(\frac{\gamma^{n-2}-\delta^{n-2}}{\gamma-\delta}-\frac{\alpha^{n-2}-\beta^{n-2}}{\alpha-\beta}\right)
\end{aligned}
$$




$$
\begin{aligned}
& +\frac{1}{3}\left(\frac{\gamma^{n+2}-\delta^{n+2}}{\gamma-\delta}-\frac{\alpha^{n+2}-\beta^{n+2}}{\alpha-\beta}\right) \\
= & \frac{1}{3(\gamma-\delta)}\left[\gamma^{n}\left(\frac{6}{\gamma}+\frac{1}{\gamma^{2}}+\gamma^{2}\right)+\delta^{n}\left(\frac{-6}{\delta}-\frac{1}{\delta^{2}}-\delta^{2}\right)\right] \\
& +\frac{1}{3(\alpha-\beta)}\left[\alpha^{n}\left(\frac{-6}{\alpha}-\frac{1}{\alpha^{2}}-\alpha^{2}\right)+\beta^{n}\left(\frac{6}{\beta}+\frac{1}{\beta^{2}}+\beta^{2}\right)\right] \\
= & \left(\gamma^{n}+\delta^{n}\right)-\left(\alpha^{n}+\beta^{n}\right),
\end{aligned}
$$

since $\frac{6}{\gamma}+\frac{1}{\gamma^{2}}+\gamma^{2}=\frac{-6}{\delta}-\frac{1}{\delta^{2}}-\delta^{2}=6 \sqrt{2}$ and $\frac{-6}{\alpha}-\frac{1}{\alpha^{2}}-\alpha^{2}=\frac{6}{\beta}+\frac{1}{\beta^{2}}+\beta^{2}=-3 \sqrt{5}$.

(3) Notice that $F_{n}=\frac{\alpha^{n}-\beta^{n}}{\alpha-\beta}$ and $P_{n}=\frac{\gamma^{n}-\delta^{n}}{\gamma-\delta}$. So we get $\sqrt{5} F_{n}=\alpha^{n}-\beta^{n}$ and $2 \sqrt{2} P_{n}=\gamma^{n}-\delta^{n}$. Thus clearly, $\sqrt{5} F_{n}+2 \sqrt{2} P_{n}=\left(\gamma^{n}-\delta^{n}\right)+\left(\alpha^{n}-\beta^{n}\right)$.

(4) It is easily seen that $P_{n+1}+P_{n-1}=\gamma^{n}+\delta^{n}$. Also $L_{n}=\alpha^{n}+\beta^{n}$. So $L_{n}+P_{n+1}+P_{n-1}=$ $\alpha^{n}+\beta^{n}+\gamma^{n}+\delta^{n}$.

(5) Since $W_{n+1}=3 W_{n}-3 W_{n-2}-W_{n-3}$, we easily get

$$
\begin{aligned}
W_{n+1}-W_{n}= & 2 W_{n}-3 W_{n-2}-W_{n-3} \\
= & 2\left(\frac{\gamma^{n}-\delta^{n}}{\gamma-\delta}-\frac{\alpha^{n}-\beta^{n}}{\alpha-\beta}\right)-3\left(\frac{\gamma^{n-2}-\delta^{n-2}}{\gamma-\delta}-\frac{\alpha^{n-2}-\beta^{n-2}}{\alpha-\beta}\right) \\
& -\left(\frac{\gamma^{n-3}-\delta^{n-3}}{\gamma-\delta}-\frac{\alpha^{n-3}-\beta^{n-3}}{\alpha-\beta}\right) \\
= & \frac{1}{\gamma-\delta}\left[\gamma^{n}\left(2-\frac{3}{\gamma^{2}}-\frac{1}{\gamma^{3}}\right)+\delta^{n}\left(-2+\frac{3}{\delta^{2}}+\frac{1}{\delta^{3}}\right)\right] \\
& +\frac{1}{\alpha-\beta}\left[\alpha^{n-1}\left(2 \alpha-\frac{3}{\alpha}-\frac{1}{\alpha^{2}}\right)-\beta^{n-1}\left(2 \beta-\frac{3}{\beta}-\frac{1}{\beta^{2}}\right)\right]
\end{aligned}
$$

and hence

$$
\begin{aligned}
& 2 W_{n+1}-2 W_{n}= \frac{2}{2 \sqrt{2}}\left[\gamma^{n}\left(\frac{2 \gamma^{3}-3 \gamma-1}{\gamma^{3}}\right)+\delta^{n}\left(\frac{-2 \delta^{2}+3 \delta+1}{\delta^{3}}\right)\right] \\
&-\frac{2}{\alpha-\beta}\left[\alpha^{n-1}\left(\frac{2 \alpha^{3}-3 \alpha-1}{\alpha^{2}}\right)-\beta^{n-1}\left(\frac{2 \beta^{3}-3 \beta-1}{\beta^{2}}\right)\right] \\
& \Leftrightarrow \quad 2 W_{n+1}- 2 W_{n}+\frac{2}{\alpha-\beta}\left[\alpha^{n-1}\left(\frac{2 \alpha^{3}-3 \alpha-1}{\alpha^{2}}\right)-\beta^{n-1}\left(\frac{2 \beta^{3}-3 \beta-1}{\beta^{2}}\right)\right] \\
&= \frac{1}{\sqrt{2}}\left[\gamma^{n}\left(\frac{2 \gamma^{3}-3 \gamma-1}{\gamma^{3}}\right)+\delta^{n}\left(\frac{-2 \delta^{3}+3 \delta+1}{\delta^{3}}\right)\right] \\
& \Leftrightarrow \quad 2\left(W_{n+1}-W_{n}+F_{n-1}\right)=\gamma^{n}+\delta^{n},
\end{aligned}
$$

since $\frac{2 \gamma^{3}-3 \gamma-1}{\gamma^{3}}=\frac{-2 \delta^{3}+3 \delta+1}{\delta^{3}}=\sqrt{2}$ and $\frac{2 \alpha^{3}-3 \alpha-1}{\alpha^{2}}=\frac{2 \beta^{3}-3 \beta-1}{\beta^{2}}=1$.

(6) It is just an algebraic computation, since $W_{n}=\left(\frac{\gamma^{n}-\delta^{n}}{\gamma-\delta}\right)-\left(\frac{\alpha^{n}-\beta^{n}}{\alpha-\beta}\right)$.

Theorem 4 The sum of the first $n$ terms of $W_{n}$ is

$$
\sum_{i=1}^{n} W_{i}=\frac{W_{n}+4 W_{n-1}+4 W_{n-2}+W_{n-3}+1}{2}
$$

for $n \geq 3$. 
Proof Recall that $W_{n}=3 W_{n-1}-3 W_{n-3}-W_{n-4}$. So

$$
W_{n-3}+W_{n-4}=3 W_{n-1}-2 W_{n-3}-W_{n}
$$

Applying (9), we deduce that

$$
\begin{aligned}
& W_{1}+W_{0}=3 W_{3}-2 W_{1}-W_{4}, \\
& W_{2}+W_{1}=3 W_{4}-2 W_{2}-W_{5}, \\
& W_{3}+W_{2}=3 W_{5}-2 W_{3}-W_{6}, \\
& \cdots, \\
& W_{n-4}+W_{n-5}=3 W_{n-2}-2 W_{n-4}-W_{n-1}, \\
& W_{n-3}+W_{n-4}=3 W_{n-1}-2 W_{n-3}-W_{n} .
\end{aligned}
$$

If we sum of both sides of (10), then we obtain $W_{n-3}+W_{0}+2\left(W_{1}+\cdots+W_{n-4}\right)=3\left(W_{3}+\right.$ $\left.W_{4}+\cdots+W_{n-1}\right)-2\left(W_{1}+W_{2}+\cdots+W_{n-3}\right)-\left(W_{4}+W_{5}+\cdots+W_{n}\right)$. So we get $W_{n-3}+2\left(W_{1}+\right.$ $\left.W_{2}+\cdots+W_{n-4}\right)=1-W_{n-2}-W_{n-1}-W_{n}+3 W_{n-2}+3 W_{n-1}$ and hence we get the desired result.

Theorem 5 The recurrence relations are

$$
\begin{aligned}
& W_{2 n}=9 W_{2 n-2}-20 W_{2 n-4}+9 W_{2 n-6}-W_{2 n-8} \\
& W_{2 n+1}=9 W_{2 n-1}-20 W_{2 n-3}+9 W_{2 n-5}-W_{2 n-7}
\end{aligned}
$$

for $n \geq 4$.

Proof Recall that $W_{n}=3 W_{n-1}-3 W_{n-3}-W_{n-4}$. So $W_{2 n}=3 W_{2 n-1}-3 W_{2 n-3}-W_{2 n-4}$ and hence

$$
\begin{aligned}
W_{2 n}= & 3 W_{2 n-1}-3 W_{2 n-3}-W_{2 n-4} \\
= & 9 W_{2 n-2}-9 W_{2 n-4}-3 W_{2 n-5}-9 W_{2 n-4}+9 W_{2 n-6}+3 W_{2 n-7} \\
& +W_{2 n-8}-W_{2 n-8}-W_{2 n-4} \\
= & -\left(3 W_{2 n-5}-3 W_{2 n-7}-W_{2 n-8}\right)+9 W_{2 n-2}-18 W_{2 n-4}+9 W_{2 n-6} \\
& -W_{2 n-8}-W_{2 n-4} \\
= & -W_{2 n-4}+9 W_{2 n-2}-9 W_{2 n-4}-9 W_{2 n-4}+9 W_{2 n-6}-W_{2 n-8}-W_{2 n-4} \\
= & 9 W_{2 n-2}-20 W_{2 n-4}+9 W_{2 n-6}-W_{2 n-8} .
\end{aligned}
$$

The other assertion can be proved similarly.

The rank of an integer $N$ is defined to be

$$
\rho(N)= \begin{cases}p & \text { if } p \text { is the smallest prime with } p \mid N \\ \infty & \text { if } N \text { is prime. }\end{cases}
$$

Thus we can give the following theorem. 
Theorem 6 The rank of $W_{n}$ is

$$
\rho\left(W_{n}\right)= \begin{cases}2 & \text { if } n=5+6 k, 6+6 k, 7+6 k \\ 3 & \text { if } n=8+12 k, 9+12 k, 15+12 k, 16+12 k \\ 5 & \text { if } n=14+60 k, 46+60 k\end{cases}
$$

for an integer $k \geq 0$.

Proof Let $n=5+6 k$. We prove it by induction on $k$. Let $k=0$. Then we get $W_{5}=24=2^{3} \cdot 3$. So $\rho\left(W_{5}\right)=2$. Let us assume that the rank of $W_{n}$ is 2 for $n=k-1$, that is, $\rho\left(W_{6 k-1}\right)=2$, so $W_{5+6(k-1)}=W_{6 k-1}=2^{a} \cdot B$ for some integers $a \geq 1$ and $B>0$. For $n=k$, we get

$$
\begin{aligned}
W_{6 k+5} & =3 W_{6 k+4}-3 W_{6 k+2}-W_{6 k+1} \\
& =3\left(3 W_{6 k+3}-3 W_{6 k+1}-W_{6 k}\right)-3 W_{6 k+2}-W_{6 k+1} \\
& =9 W_{6 k+3}-9 W_{6 k+1}-3 W_{6 k}-3 W_{6 k+2}-W_{6 k+1} \\
& =9\left(3 W_{6 k+2}-3 W_{6 k}-W_{6 k-1}\right)-9 W_{6 k+1}-3 W_{6 k}-3 W_{6 k+2}-W_{6 k+1} \\
& =27 W_{6 k+2}-27 W_{6 k}-9 W_{6 k-1}-9 W_{6 k+1}-3 W_{6 k}-3 W_{6 k+2}-W_{6 k+1} \\
& =24 W_{6 k+2}-30 W_{6 k}-10 W_{6 k+1}-9 W_{6 k-1} \\
& =24 W_{6 k+2}-30 W_{6 k}-10 W_{6 k+1}-9 \cdot 2^{a} B \\
& =2\left[12 W_{6 k+2}-15 W_{6 k}-5 W_{6 k+1}-9 \cdot 2^{a-1} B\right] .
\end{aligned}
$$

Therefore $\rho\left(W_{5+6 k}\right)=2$. Similarly it can be shown that $\rho\left(W_{6+6 k}\right)=\rho\left(W_{7+6 k}\right)=2$.

Now let $n=8+12 k$. For $k=0$, we get $W_{8}=387=3^{2} \cdot 43$. So $\rho\left(W_{8}\right)=3$. Let us assume that for $n=k-1$ the rank of $W_{n}$ is 3 , that is, $\rho\left(W_{8+12(k-1)}\right)=\rho\left(W_{12 k-4}\right)=3^{b} \cdot H$ for some integers $b \geq 1$ and $H>0$ which is not even integer. For $n=k$, we get

$$
\begin{aligned}
W_{12 k+8}= & 3 W_{12 k+7}-3 W_{12 k+5}-W_{12 k+4} \\
= & 3 W_{12 k+7}-3 W_{12 k+5}-\left(3 W_{12 k+3}-3 W_{12 k+1}-W_{12 k}\right) \\
= & 3 W_{12 k+7}-3 W_{12 k+5}-3 W_{12 k+3}+3 W_{12 k+1}+W_{12 k} \\
= & 3 W_{12 k+7}-3 W_{12 k+5}-3 W_{12 k+3}+3 W_{12 k+1} \\
& +\left(3 W_{12 k-1}-3 W_{12 k-3}-W_{12 k-4}\right) \\
= & 3 W_{12 k+7}-3 W_{12 k+5}-3 W_{12 k+3}+3 W_{12 k+1}+3 W_{12 k-1} \\
& -3 W_{12 k-3}-W_{12 k-4} \\
= & 3 W_{12 k+7}-3 W_{12 k+5}-3 W_{12 k+3}+3 W_{12 k+1}+3 W_{12 k-1} \\
& -3 W_{12 k-3}-3^{b} \cdot H \\
= & 3\left(W_{12 k+7}-W_{12 k+5}-W_{12 k+3}+W_{12 k+1}+W_{12 k-1}\right. \\
& \left.-W_{12 k-3}-3^{b-1} \cdot H\right) .
\end{aligned}
$$

So $\rho\left(W_{12 k+8}\right)=3$. The others can be proved similarly. 
Remark 1 Apart from the above theorem, we see that $\rho\left(W_{22}\right)=\rho\left(W_{26}\right)=\infty$, while $\rho\left(W_{70}\right)=\rho\left(W_{98}\right)=13$ and $\rho\left(W_{10}\right)=\rho\left(W_{34}\right)=\rho\left(W_{50}\right)=23$. But there is no general formula.

The companion matrix for $W_{n}$ is

$$
M=\left[\begin{array}{cccc}
3 & 0 & -3 & -1 \\
1 & 0 & 0 & 0 \\
0 & 1 & 0 & 0 \\
0 & 0 & 1 & 0
\end{array}\right]
$$

Set

$$
N=\left[\begin{array}{l}
1 \\
0 \\
0 \\
0
\end{array}\right]
$$

and

$$
R=\left[\begin{array}{llll}
3 & 1 & 0 & 0
\end{array}\right]
$$

Then we can give the following theorem, which can be proved by induction on $n$.

Theorem 7 For the sequence $W_{n}$, we have:

(1) $R M^{n} N=W_{n+3}+P_{n}+2\left(W_{n+1}-F_{n}\right)$ for $n \geq 1$.

(2) $R\left(M^{T}\right)^{n-3} N=W_{n}$ for $n \geq 3$.

(3) If $n \geq 7$ is odd, then

$$
M^{n}=\left[\begin{array}{llll}
m_{11} & m_{12} & m_{13} & m_{14} \\
m_{21} & m_{22} & m_{23} & m_{24} \\
m_{31} & m_{32} & m_{33} & m_{34} \\
m_{41} & m_{42} & m_{43} & m_{44}
\end{array}\right]
$$

where

$$
\begin{aligned}
& m_{11}=W_{n+2}, \quad m_{21}=W_{n+1}, \quad m_{31}=W_{n}, \quad m_{41}=W_{n-1}, \\
& m_{14}=-W_{n+1}, \quad m_{24}=-W_{n}, \quad m_{34}=-W_{n-1}, \quad m_{44}=-W_{n-2}, \\
& m_{12}=-1-W_{n+1}-2 \sum_{i=0}^{\frac{n-5}{2}} W_{n-1-2 i}, \quad m_{13}=-W_{n+2}-2 \sum_{i=0}^{\frac{n-3}{2}} W_{n-2 i}, \\
& m_{22}=-W_{n}-2 \sum_{i=0}^{\frac{n-5}{2}} W_{n-2-2 i}, \quad m_{23}=-1-W_{n+1}-2 \sum_{i=0}^{\frac{n-5}{2}} W_{n-1-2 i}, \\
& m_{32}=-1-W_{n-1}-2 \sum_{i=0}^{\frac{n-7}{2}} W_{n-3-2 i}, \quad m_{33}=-W_{n}-2 \sum_{i=0}^{\frac{n-5}{2}} W_{n-2-2 i},
\end{aligned}
$$




$$
m_{42}=-W_{n-2}-2 \sum_{i=0}^{\frac{n-7}{2}} W_{n-4-2 i}, \quad m_{43}=-1-W_{n-1}-2 \sum_{i=0}^{\frac{n-7}{2}} W_{n-3-2 i}
$$

and if $n \geq 8$ is even, then

$$
M^{n}=\left[\begin{array}{llll}
m_{11} & m_{12} & m_{13} & m_{14} \\
m_{21} & m_{22} & m_{23} & m_{24} \\
m_{31} & m_{32} & m_{33} & m_{34} \\
m_{41} & m_{42} & m_{43} & m_{44}
\end{array}\right],
$$

where

$$
\begin{aligned}
& m_{11}=W_{n+2}, \quad m_{21}=W_{n+1}, \quad m_{31}=W_{n}, \quad m_{41}=W_{n-1}, \\
& m_{14}=-W_{n+1}, \quad m_{24}=-W_{n}, \quad m_{34}=-W_{n-1}, \quad m_{44}=-W_{n-2}, \\
& m_{12}=-W_{n+1}-2 \sum_{i=0}^{\frac{n-4}{2}} W_{n-1-2 i}, \quad m_{13}=-1-W_{n+2}-2 \sum_{i=0}^{\frac{n-4}{2}} W_{n-2 i}, \\
& m_{22}=-1-W_{n}-2 \sum_{i=0}^{\frac{n-6}{2}} W_{n-2-2 i}, \quad m_{23}=-W_{n+1}-2 \sum_{i=0}^{\frac{n-4}{2}} W_{n-1-2 i}, \\
& m_{32}=-W_{n-1}-2 \sum_{i=0}^{\frac{n-6}{2}} W_{n-3-2 i}, \quad m_{33}=-1-W_{n}-2 \sum_{i=0}^{\frac{n-6}{2}} W_{n-2-2 i}, \\
& m_{42}=-1-W_{n-2}-2 \sum_{i=0}^{\frac{n-8}{2}} W_{n-4-2 i},
\end{aligned}
$$

A circulant matrix is a matrix $A=\left[a_{i j}\right]_{n \times n}$ defined to be

$$
A=\left[\begin{array}{ccccc}
a_{0} & a_{1} & a_{2} & \cdots & a_{n-1} \\
a_{n-1} & a_{0} & a_{1} & \cdots & a_{n-2} \\
a_{n-2} & a_{n-1} & a_{0} & \cdots & a_{n-3} \\
\cdot & \cdot & \cdot & \cdots & \cdot \\
\cdot & \cdot & \cdot & \cdots & \cdot \\
a_{1} & a_{2} & a_{3} & \cdots & a_{0}
\end{array}\right]
$$

where $a_{i}$ are constants. The eigenvalues of $A$ are

$$
\lambda_{j}(A)=\sum_{k=0}^{n-1} a_{k} w^{-j k}
$$

where $w=e^{\frac{2 \pi i}{n}}, i=\sqrt{-1}$, and $j=0,1, \ldots, n-1$. The spectral norm for a matrix $B=\left[b_{i j}\right]_{n \times m}$ is defined to be $\|B\|_{\text {spec }}=\max \left\{\sqrt{\lambda_{i}}\right\}$, where $\lambda_{i}$ are the eigenvalues of $B^{H} B$ for $0 \leq j \leq n-1$ and $B^{H}$ denotes the conjugate transpose of $B$. 
For the circulant matrix

$$
W=W\left(W_{n}\right)=\left[\begin{array}{ccccc}
W_{0} & W_{1} & W_{2} & \cdots & W_{n-1} \\
W_{n-1} & W_{0} & W_{1} & \cdots & W_{n-2} \\
W_{n-2} & W_{n-1} & W_{0} & \cdots & W_{n-3} \\
\cdot & \cdot & \cdot & \cdots & \cdot \\
\cdot & \cdot & \cdot & \cdots & \cdot \\
W_{1} & W_{2} & W_{3} & \cdots & W_{0}
\end{array}\right]
$$

for $W_{n}$, we can give the following theorem.

\section{Theorem 8 The eigenvalues of $W$ are}

$$
\lambda_{j}(W)=\frac{\left\{\begin{array}{c}
W_{n-1} w^{-3 j}+\left(W_{n}+P_{n-1}-2 F_{n-1}+1\right) w^{-2 j} \\
+\left(P_{n}-2 F_{n}-W_{n-1}\right) w^{-j}-W_{n}
\end{array}\right\}}{w^{-4 j}+3 w^{-3 j}-3 w^{-j}+1}
$$

for $j=0,1,2, \ldots, n-1$.

Proof Applying (11) we easily get

$$
\begin{aligned}
\lambda_{j}(W)= & \sum_{k=0}^{n-1} W_{k} w^{-j k}=\sum_{k=0}^{n-1}\left(\frac{\gamma^{k}-\delta^{k}}{\gamma-\delta}-\frac{\alpha^{k}-\beta^{k}}{\alpha-\beta}\right) w^{-j k} \\
= & \frac{1}{\gamma-\delta}\left[\frac{\gamma^{n}-1}{\gamma w^{-j}-1}-\frac{\delta^{n}-1}{\delta w^{-j}-1}\right]-\frac{1}{\alpha-\beta}\left[\frac{\alpha^{n}-1}{\alpha w^{-j}-1}-\frac{\beta^{n}-1}{\beta w^{-j}-1}\right] \\
= & \frac{1}{\gamma-\delta}\left[\frac{\left(\gamma^{n}-1\right)\left(\delta w^{-j}-1\right)-\left(\delta^{n}-1\right)\left(\gamma w^{-j}-1\right)}{\left(\gamma w^{-j}-1\right)\left(\delta w^{-j}-1\right)}\right] \\
& -\frac{1}{\alpha-\beta}\left[\frac{\left(\alpha^{n}-1\right)\left(\beta w^{-j}-1\right)-\left(\beta^{n}-1\right)\left(\alpha w^{-j}-1\right)}{\left(\alpha w^{-j}-1\right)\left(\beta w^{-j}-1\right)}\right] \\
= & \frac{1}{\gamma-\delta}\left[\frac{w^{-j}\left(\gamma^{n} \delta-\delta^{n} \gamma+\gamma-\delta\right)+\delta^{n}-\gamma^{n}}{\delta \gamma w^{-2 j}-w^{-j}(\delta+\gamma)+1}\right] \\
& -\frac{1}{\alpha-\beta}\left[\frac{w^{-j}\left(\alpha^{n} \beta-\beta^{n} \alpha+\alpha-\beta\right)+\beta^{n}-\alpha^{n}}{\beta \alpha w^{-2 j}-w^{-j}(\beta+\alpha)+1}\right] \\
= & \left.\begin{array}{c}
w^{-3 j}\left[\sqrt{5}\left(\delta-\gamma+\gamma \delta^{n}-\delta \gamma^{n}\right)+2 \sqrt{2}\left(\alpha-\beta+\alpha^{n} \beta-\alpha \beta^{n}\right)\right] \\
+w^{-2 j}\left[\sqrt{5}\left(\gamma^{n}-\delta^{n}+\delta-\gamma+\gamma \delta^{n}-\gamma^{n} \delta\right)+2 \sqrt{2}\left(\beta^{n}-\alpha^{n}\right)\right. \\
\left.+4 \sqrt{2}\left(\alpha-\beta+\alpha^{n} \beta-\alpha \beta^{n}\right)\right]+w^{-j}\left[\sqrt { 5 } \left(\gamma^{n}-\delta^{n}+\gamma-\delta\right.\right. \\
\left.\left.+\gamma^{n} \delta-\gamma \delta^{n}\right)+2 \sqrt{2}\left(\beta-\alpha+\beta^{n} \alpha-\alpha^{n} \beta\right)+4 \sqrt{2}\left(\beta^{n}-\alpha^{n}\right)\right] \\
+\left[\sqrt{5}\left(\delta^{n}-\gamma^{n}\right)+2 \sqrt{2}\left(\alpha^{n}-\beta^{n}\right)\right] \\
2 \sqrt{10}\left(w^{-4 j}+3 w^{-3 j}-3 w^{-j}+1\right)
\end{array}\right\} \\
=\left\{\begin{array}{c}
W_{n-1} w^{-3 j}+\left(W_{n}+P_{n-1}-2 F_{n-1}+1\right) w^{-2 j} \\
+\left(P_{n}-2 F_{n}-W_{n-1}\right) w^{-j}-W_{n}
\end{array}\right\} & \frac{w^{-4 j}+3 w^{-3 j}-3 w^{-j}+1}{2},
\end{aligned}
$$

since $\alpha \beta=-1, \gamma \delta=-1, \alpha+\beta=1, \alpha-\beta=\sqrt{5}, \gamma+\delta=2$, and $\gamma-\delta=2 \sqrt{2}$. 
After all, we consider the spectral norm of $W$. Let $n=2$. Then $W_{2}=[0]_{2 \times 2}$. So $\left\|W_{2}\right\|_{\text {spec }}=0$. Similarly for $n=3$, we get

$$
W_{3}=\left[\begin{array}{lll}
0 & 0 & 1 \\
1 & 0 & 0 \\
0 & 1 & 0
\end{array}\right]
$$

and hence $W_{3}^{H} W_{3}=I_{3}$. So $\left\|W_{3}\right\|_{\text {spec }}=1$. For $n \geq 4$, the spectral norm of $W_{n}$ is given by the following theorem, which can be proved by induction on $n$.

Theorem 9 The spectral norm of $W_{n}$ is

$$
\left\|W_{n}\right\|_{\text {spec }}=\frac{W_{n-1}+4 W_{n-2}+4 W_{n-3}+W_{n-4}+1}{2}
$$

for $n \geq 4$.

For example, let $n=6$. Then the eigenvalues of $W_{6}^{H} W_{6}$ are

$$
\lambda_{0}=1,369, \quad \lambda_{1}=289, \quad \lambda_{2}=\lambda_{4}=784 \quad \text { and } \quad \lambda_{3}=\lambda_{5}=388 .
$$

So the spectral norm is $\left\|W_{6}\right\|_{\text {spec }}=\sqrt{\lambda_{0}}=37$. Also $\frac{W_{5}+4 W_{4}+4 W_{3}+W_{2}+1}{2}=37$. Consequently,

$$
\left\|W_{6}\right\|_{\text {spec }}=\frac{W_{5}+4 W_{4}+4 W_{3}+W_{2}+1}{2}=37
$$

as we claimed.

\section{Competing interests}

The author declares that they have no competing interests.

\section{Acknowledgements}

The author wishes to thank Professor Ahmet Tekcan of Uludag University for constructive suggestions.

Received: 25 March 2015 Accepted: 26 April 2015 Published online: 08 May 2015

\section{References}

1. Conway, JH, Guy, RK: Fibonacci numbers. In: The Book of Numbers. Springer, New York (1996)

2. Hilton, P, Holton, D, Pedersen, J: Fibonacci and Lucas numbers. In: Mathematical Reflections in a Room with Many Mirrors, Chapter 3. Springer, New York (1997)

3. Koshy, T: Fibonacci and Lucas Numbers with Applications. Wiley, New York (2001)

4. Niven, I, Zuckerman, HS, Montgomery, HL: An Introduction to the Theory of Numbers, 5th edn. Wiley, New York (1991)

5. Ogilvy, CS, Anderson, JT: Fibonacci numbers. In: Excursions in Number Theory, Chapter 11. Dover, New York (1988)

6. Ribenboim, P: My Numbers, My Friends: Popular Lectures on Number Theory. Springer, New York (2000)

7. TaşCI, D: On quadrapell numbers and quadrapell polynomials. Hacet. J. Math. Stat. 38(3), 265-275 (2009)

8. Tekcan, A, Özkoç, A, Engür, M, Özbek, ME: On algebraic identities on a new integer sequence with four parameters. Ars Comb. (accepted) 